\title{
Grouping with and without attention
}

\author{
WAI YEN CHAN and FOOK K. CHUA \\ National University of Singapore, Singapore
}

\begin{abstract}
The effects of perceptual grouping on a line discrimination task were investigated using Moore and Egeth's (1997) paradigm. Observers judged which of two lines, presented one above the other over a matrix of spots, was longer. On some trials, larger spots at both ends of the lines formed arrowheads, thereby making possible the Müller-Lyer illusion. When observers attended only to the lines, they were not aware of the arrowheads. Yet their line judgment performance showed that they had succumbed to the illusion. When the observers' attention was directed to the arrowheads but they nevertheless failed to discern the arrowheads' orientation, their line judgments were still influenced by the illusion.
\end{abstract}

The question addressed in this report is whether grouping occurs preattentively (e.g., Treisman, 1986) or postattentively (e.g., Barchilon Ben-Av, Sagi, \& Braun, 1992; Mack, Tang, Tuma, Kahn, \& Rock, 1992). In one commonly used method (e.g., Barchilon Ben-Av et al., 1992), the effects that obtained under divided attention, where the grouping operation was the secondary task, are compared with those that obtained when it was the main or the sole task (full attention). If the grouped patterns were harder to discern under the divided-attention condition, the interpretation is that the grouping operation demanded attentional resources and, thus, could not have occurred preattentively. Mack et al. raised an important methodological point. Forewarning observers that the background elements could be grouped may influence the perceptual strategies that they use. Thus, even if the two conditions yielded comparable results, one cannot be certain that the same grouping effects would also have obtained if attention had not been explicitly deployed to the elements making up the group.

The inattention paradigm introduced by Mack et al. (1992) circumvents this problem. Observers were required to perform a demanding task, such as discriminating the lengths of two lines that differed by a small amount. In one version of the task, the lines were overlaid on a homogeneous background. On a trial toward the end of the experiment, a small number of background elements were made dissimilar to the rest so that, if they were grouped, one quadrant appeared to be segregated from the rest. After the line judgment response, the participants were unexpectedly required to locate the errant quadrant. They generally failed in this task. The main line judgment task required focused attention. Mack

This research was supported by Grant R-107-000-013-112from the National University of Singapore. W.Y.C. gathered the data under the supervision of F.K.C. in partial fulfillment of a BA degree. Correspondence concerning this article should be sent to F. K. Chua, Department of Social Work and Psychology, National University of Singapore, Kent Ridge Crescent, Singapore 119260 (e-mail: fkchua@ leonis.nus.edu.sg). et al. argued that if preattentive grouping of the dissimilar elements had occurred, observers ought to be able to locate the segregated quadrant. They concluded that grouping could not have occurred preattentively and that perceptual organization required attention.

Moore and Egeth (1997) criticized Mack et al.'s (1992) experiments on the grounds that they conflated failure to group with failure to recall. What Mack et al.'s participants failed to do was to report where the grouped elements were. This failure, however, need not imply that the elements were not grouped in the first place. For example, grouping may indeed have occurred, but the location of the grouped elements might not have been encoded, since remembering it was not explicitly demanded. As a result, the participants were unable to report the location information when they were unexpectedly asked to do so.

In order to separate the two, Moore and Egeth (1997) manipulated implicit priming in a variation of Mack et al.'s (1992) inattention paradigm. They exploited two line illusions (the Ponzo in their Experiments 1 and 2 and the Müller-Lyer [ML] in their Experiment 3). In their ML experiments, two horizontal lines of equal length were superimposed in a rectangular matrix of white spots. A small number of the spots were black. On some trials, these black spots formed arrowheads at both ends of the two lines. On one line, the arrowheads pointed in (wings out), and on the other, they pointed out (wings in; we adopted this terminology), thus creating the ML display. The question they asked was whether the ML illusion occurred even when the observers were engaged in the demanding line judgment task. They reasoned that if the illusion occurred (i.e., the participants reported the line enclosed by the wings-in arrowheads as longer) even when the participants failed to report seeing the arrowheads, it must be the case that grouping of the black spots obtained preattentively. Their results showed that their participants were not aware of the arrowheads but consistently judged that the line enclosed by the wings-in arrowheads was longer, implying that there had been preattentive grouping. The effects of grouping and the contents of 
the grouping operation thus appear to have been dissociated. Recent demonstrations of the fragility of information that is not immediately encoded (see, e.g., the volume edited by Coltheart, 1999) are consistent with this account. What Moore and Egeth appear to have been arguing is that what had been classified as inattentional blindness by Mack et al. (1992) was, in fact, a case of inattentional amnesia (Wolfe, 1999).

In an earlier series of experiments in which a dividedattention paradigm was used, Barchilon Ben-Av et al. (1992) showed that the grouping operation appeared to demand attentional resources. They compared performance in a dual-task condition, in which the identification of the background pattern constituted the secondary task, with a condition in which it was the main task. In some experiments, the background elements, grouped by proximity, formed columns or rows. In other experiments, grouping was by (shape) similarity, with elements having the same shape appearing as columns or rows. Barchilon Ben-Av et al. showed that even when the grouping task merely required the detection of a grouped pattern, the observers did significantly worse when grouping was the secondary task than when they performed the task alone (their Experiments 5 and 6). However, when the grouping operation was performed as the primary task and the secondary task was one that did not require attention (singleton detection in their Experiment 4 ), there was no difference between the single- and the dual-task conditions.

There is evidence that some grouping operations are demanding and require attentional resources, whereas others can occur preattentively (Sagi \& Julesz, 1985). Recently, Barchilon Ben-Av and Sagi (1995) showed that time courses varied with different modes of grouping operations. Contrast Moore and Egeth's (1997) and Barchilon Ben-Av et al.'s (1992) experiments. Although both experiments manipulated grouping by similarity, Moore and Egeth used luminance, whereas Barchilon Ben-Av et al. used shape as the grouping variable. A prima facie case may be made that grouping by shape (with orientation of the shapes randomly perturbed) in Barchilon Ben-Av et al.'s displays was difficult. Thus, it is possible that Moore and Egeth found preattentive grouping because luminance differences allowed grouping to occur swiftly. We picked up these leads in this experiment: Grouping difficulty was manipulated as a main variable.

The task used was similar to Moore and Egeth's (1997) Experiment 3. Participants were asked to judge which of two lines, presented one above the other, was longer. The lines were superimposed on a matrix of spots, some of which were larger. In the critical trials, the larger spots formed arrowheads on both ends of the lines. If grouping by size (similarity) occurred preattentively, the larger spots would be perceived as wings-out and wings-in arrowheads, and line judgment would be affected accordingly.

Two variables, both likely to affect ease of grouping by size, were manipulated. First, the relative sizes of the background spots and those making up the arrowheads were varied. A large difference in size makes the arrow- heads more apparent. Second, on half the trials, the spots were aligned, forming a square grid. For the rest, the positions of the spots were randomly jittered, disrupting the grid pattern and, also, the alignment of spots forming the arrowheads. This should make grouping more difficult, and thus the ML illusion ought to be attenuated.

We made two changes to Moore and Egeth's (1997) procedure. First, whereas they presented two lines of equal length, we used lines that always differed by 4 pixels. We contrasted performance when the ML arrowheads were presented (ML condition) and when they were not (baseline condition). The baseline condition allowed us to assess how well the participants were able to resolve this 4-pixel difference. In the ML condition, the wings-out arrowheads were always presented with the shorter line. Thus, if the participants succumbed to the illusion, they would report that the shorter line was longer more often than they would in the baseline condition. The difference between the baseline and the ML conditions was used to gauge the strength of the illusion. The second difference was that the participants went through several blocks of practice trials in which the ML arrowheads were not presented. The purpose was twofold: to ensure that the participants were practiced on the line discrimination task and to get them accustomed to the background so that they could focus exclusively on the lines and filter out the spots.

\section{METHOD}

\section{Participants}

The observers were 14 volunteers from the undergraduate pool, all of whom had normal or corrected-to-normal vision. The data from 1 participant were excluded, since he misunderstood the instructions.

\section{Stimuli and Design}

The experiment contained three parts. In the first part (inattention), the participants were required to perform only the line discrimination task. In the second part (divided attention), they were required to perform the line discrimination task as the primary task and then to report the orientation of the arrowheads as the secondary task. In the final part (full attention), they were told to ignore the lines and attend only to the arrowheads. The participants were briefed about the task requirements of the different parts of the experiment only when that part began.

On each trial, a pair of lines was presented on a matrix of $24 \times$ 24 spots (subtending a visual angle of $16^{\circ} \times 16^{\circ}$ ). The two lines differed by 4 pixels (visual angle $\approx 0.14^{\circ}$ ), with the shorter line being 156 pixels long. As has been mentioned, the wings-in arrowheads were always presented with the shorter line, and the wings-out arrowheads were presented with the longer line. The two lines were separated vertically by 140 pixels (visual angle $\approx 5^{\circ}$ ). The longer line (160 pixels) was presented on the top for half the trials. There were two types of display matrices. In the ML condition, 7 spots at either end of a line were larger than the background spots, so that the line appeared to be enclosed by arrowheads (see Figure 1A). In the inattention blocks, the baseline condition consisted of presenting an equal number of larger spots in random locations around the two ends of the lines (Figure 1B). For the divided- and full-attention blocks, the baseline condition consisted of enclosing the lines with identical arrowheads (both wings in or both wings out).

There were two main variables: disparity in size between the ML and the background spots (the disparity variable) and the alignment of the spots in the display (the jitter variable). The diameter of the spots forming the arrowheads was always 12 pixels. There were three 
A

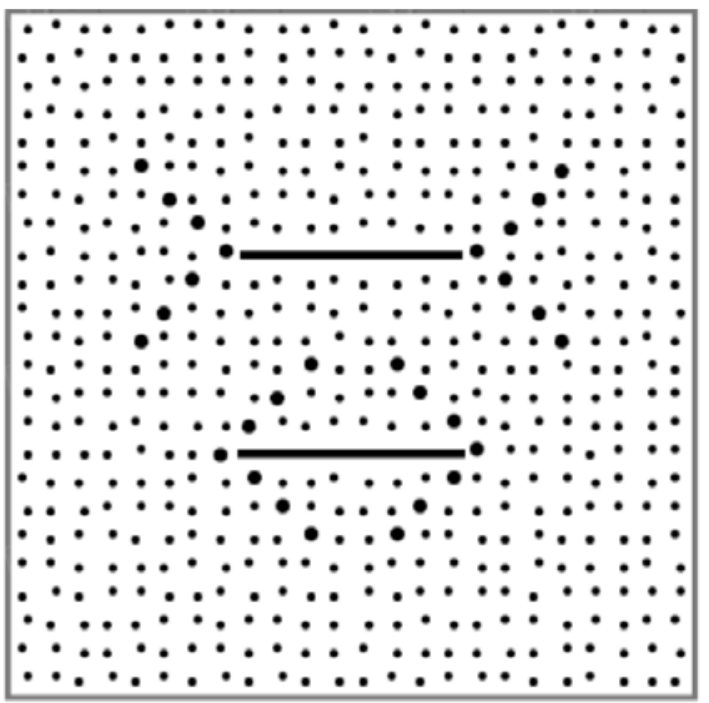

B

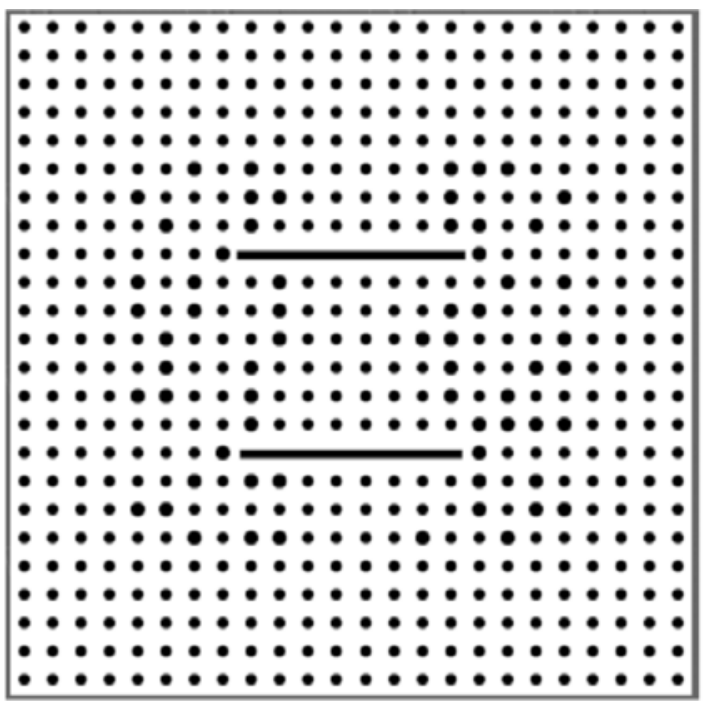

Figure 1. (A) An example of a large-disparity jittered display, Müller-Lyer trials. (B) An example of a medium-disparity aligned display, baseline trials.

background spot sizes (diameter $=6,8$, or 10 pixels), and the difference between them and the ML spots formed, respectively, the large(6 pixels), medium- (4 pixels), and small- (2 pixels) disparity conditions. On half the trials, all the spots were perfectly aligned to form a grid pattern (aligned condition, Figure 1B). For the rest of the trials, the grid pattern was disrupted by shifting the spots randomly by up to 2 pixels in both $x$ - and $y$-directions (jittered condition, Figure 1A).

\section{Procedure}

Each trial began with a fixation cross lasting approximately $1,000 \mathrm{msec}$. The display was presented for $200 \mathrm{msec}$, followed by a random-dot mask that stayed on the screen until the observers entered their response via a dialog box.

For the inattention blocks, the participants were required only to judge whether the top or the bottom line was longer. There were 10 blocks, with the first 5 designated as practice. (The observers were not told that these were practice trials.) In these blocks, only the baseline displays (i.e., no arrowheads) were presented. On half of these practice trials, the difference between the two lines was longer ( 6 pixels, $0.2^{\circ}$ ). There were 24 trials in each block, obtained by factorially crossing the pixel difference ( 4 or 6 pixels), jittering, size disparity, and position of the longer line variables. Feedback (tone on error) was provided only for these 5 practice blocks. In the next 5 experimental blocks, 12 additional ML trials (jittering, size disparity, and position of longer line variables factorially crossed) were included. For these trials, the two lines always differed by 4 pixels. The observers were asked, at the end of the inattention blocks, whether they had seen any pattern in the displays.

In the divided-attention blocks, the observers were required first to judge whether the top or the bottom line was longer (as in the inattention blocks) and then to identify the orientation of the arrowheads that enclosed each of the two lines (i.e., whether they flanked outward or inward). They were briefed to treat line judgment as the primary task and arrowhead identification as the secondary task. There was always a 4-pixel difference between the two lines. The ML trials were identical to those in the inattention blocks. For the baseline trials, the orientation of the arrowheads that enclosed each of the two lines was identical. The trials were presented in the fashion described previously. The participants entered their responses via two dialog boxes that appeared one after the other. For arrowhead identification, there were five options: (1) wingsout top, wings-in bottom; (2) wings in, wings out; (3) both wings in; (4) both wings out; and (5) "could not see arrowheads." The participants completed four blocks, each of 24 trials.

The displays of the full-attention blocks were identical to the divided-attention blocks. Here, the participants were told to ignore the lines and to focus entirely on the arrowheads. There was a single dialog box, requiring them to report the orientation of the arrowheads, at the end of the trial. There were four blocks in this part.

\section{RESULTS AND DISCUSSION}

This section is organized as follows. First, we will compare the arrowhead identification performance in the divided-and full-attention blocks where grouping was explicitly demanded. Next, we will examine whether the arrowheads in the inattention blocks affected line judgment. Finally, we will contrast the line judgment performance between the divided-attention and the inattention blocks.

\section{Arrowhead Identification}

As has been discussed previously, comparing arrowhead identification in the full- and the divided-attention trials will not unambiguously answer the question whether preattentive grouping obtained. Nevertheless, it will provide some indication of how easy it was to perceive the arrowheads in the different display conditions and to report it while discriminating the lines. This forms the basis for evaluating the magnitude of the illusion in the inattention blocks. For example, if size disparity affected how easily and, therefore, how quickly grouping might occur, it may be expected that the strength of the illusion should increase with disparity.

In the baseline condition, the orientations of the arrowheads on both lines were identical; in the ML condition, the orientations were different. First, we established that performance in both conditions was comparable. A 
2 (display type: baseline vs. ML) $\times 2$ (attention instruction: divided vs. full attention) $\times 3$ (disparity) $\times 2$ (jitter) analysis of variance (ANOVA) showed no overall difference between the baseline and the ML trials $(F<1)$, and the display type variable did not interact with the other variables. Therefore, the data were collapsed across the display type variable for the rest of the analysis.

Performance in the arrowhead identification task as a function of the disparity and jitter variables is presented in Figure 2. In a 2 (attention instruction) $\times 3$ (disparity) $\times$ 2 (jitter) ANOVA, there was a main effect of instruction $\left[F(1,12)=53.705, M S_{\mathrm{e}}=0.042, p<.001\right]$, with performance significantly better for the full-attention blocks. There was also a main effect of disparity $[F(2,24)=$ $\left.222.320, M S_{\mathrm{e}}=0.033, p<.001\right]$. Performance was very poor in the small-disparity condition: The participants reported that they could not see any arrowheads on more than $70 \%$ of the trials. As disparity increased, their identification performance improved. The attention instruction $\times$ disparity interaction was reliable $[F(2,24)=$ $\left.31.991, M S_{\mathrm{e}}=0.022, p<.001\right]$. Simple effect comparisons showed better identification in the full-attention condition only for the medium and large disparities. The main effect of jitter was significant $[F(1,12)=28.798$, $\left.M S_{\mathrm{e}}=0.013, p<.001\right]$. When the display was aligned, performance was better. There was also a reliable disparity $\times$ jitter interaction $\left[F(1,12)=22.830, M S_{\mathrm{e}}=\right.$ $0.010, p<.001]$. The aligned display produced better performance only for the medium and large disparities.
When the disparity was large, jittering the display did not affect performance. But in the medium-disparity condition, jittering the display produced chance performance. The observers reported seeing the arrowheads on $74 \%$ of the trials. But their accuracy was only $21 \%$, suggesting that on these trials, they were more or less guessing the orientation of the arrowheads $(74 / 4=18.5 \%)$. The three-way interaction was not significant $[F(2,24)=$ $\left.1.899, M S_{\mathrm{e}}=0.011, p>.1\right]$.

In summary, these results showed that (1) arrowhead identification, the secondary task, was performed more poorly in the divided-attention trials; (2) in the smalldisparity condition, the arrowheads could not be discerned even in the full-attention condition; and (3) jittering the display made the arrowheads harder to identify primarily in the medium-disparity condition.

The poorer performance under the divided-attention condition replicated Barchilon Ben-Av et al. (1992). What is noteworthy is that poorer performance was obtained even in the large-disparity condition, in which, under full attention, performance was virtually at ceiling level, suggesting that the arrowheads were grouped rather easily. According to Barchilon Ben-Av et al., the performance difference between full and divided-attention implies that the grouping operation demands attentional resources. Now, if preattentive grouping did not occur, there should be no difference in line-judgment performance on the ML and baseline trials for the inattention condition.



Figure 2. Percentages of correct arrowhead identifications for the divided-and the full-attention blocks as a function of the background pattern. The two bars in each group represent performance in the divided- (unfilled bar) and the full- (filled bar) attention blocks. Left panel, small disparity; center panel, medium disparity; and right panel, large disparity. Error bar $=1 S E$. 


\section{Line Judgments: Inattention Condition}

For the inattention task, only the data from the five experimental blocks were analyzed (i.e., those in which the lines differed by 4 pixels). The data (correct identification of the longer line) as a function of size disparity and the jitter variables are presented in Figure 3. To the extent that the ML illusion occurred, there should be poorer performance in the ML condition than in the baseline condition. A 2 (display type: ML vs. baseline) $\times 3$ (disparity) $\times 2$ (jitter) ANOVA revealed three reliable main effects. The ML condition produced poorer performance $\left[F(1,12)=11.400, M S_{\mathrm{e}}=0.032, p<.01\right]$. As size disparity increased, performance declined $[F(2,24)=$ $\left.4.332, M S_{\mathrm{e}}=0.021, p<.05\right]$. When the display was jittered, performance also declined $[F(1,12)=5.248$, $\left.M S_{\mathrm{e}}=0.031, p<.05\right]$. The interaction between the display type and the disparity variables was significant $\left[F(2,24)=3.964, M S_{\mathrm{e}}=0.015, p<.05\right]$, suggesting that the illusion became more apparent as disparity increased. No other interactions were reliable.

Recall that in the full-attention blocks, the orientation of the arrowheads could not be seen when disparity was smallest. An analysis of the simple effects showed that there was no difference between the baseline and the ML trials at the smallest size disparity $(F<1)$. This is not surprising. When the arrowheads were nearly impossible to discern, the participants were more or less immune to the ML illusion. For the medium- and large-disparity conditions, performance was significantly worse in the
ML condition $\left[F(1,12)=24.362, M S_{\mathrm{e}}=0.013, p<\right.$ .001 , and $F(1,12)=5.455, M S_{\mathrm{e}}=0.030, p<.05$, respectively]. None of the participants reported seeing any pattern in the inattention condition. The results strongly suggest preattentive grouping, broadly replicating Moore and Egeth (1997).

Moore and Egeth (1997) argued that the failure to report did not imply a failure to group. Our finding showed something slightly different: Grouping that obtained via explicit procedures may not be the same as that which obtained when grouping was not explicitly demanded. Recall that when the background spots were medium sized, observers were able to report the orientation of the arrowheads only when the spots were aligned. When the display was jittered, performance fell to chance level. Yet line judgment was significantly worse than baseline, implying that the observers succumbed to the illusion. Thus, the fact that grouping influenced line judgment need not necessarily imply that the grouped structure could be recovered under explicit processing.

There was an unexpected finding that spoke to the dissociation between what is explicitly (full and dividedattention) and implicitly (inattention) encoded. We reasoned that the result of jittering the display would be that the orientation of the arrowhead would be encoded with less fidelity. We observed this. We further reasoned that the ML illusion would be attenuated when the spots were jittered. The data instead showed that the difference between the ML and the baseline was larger in the jittered

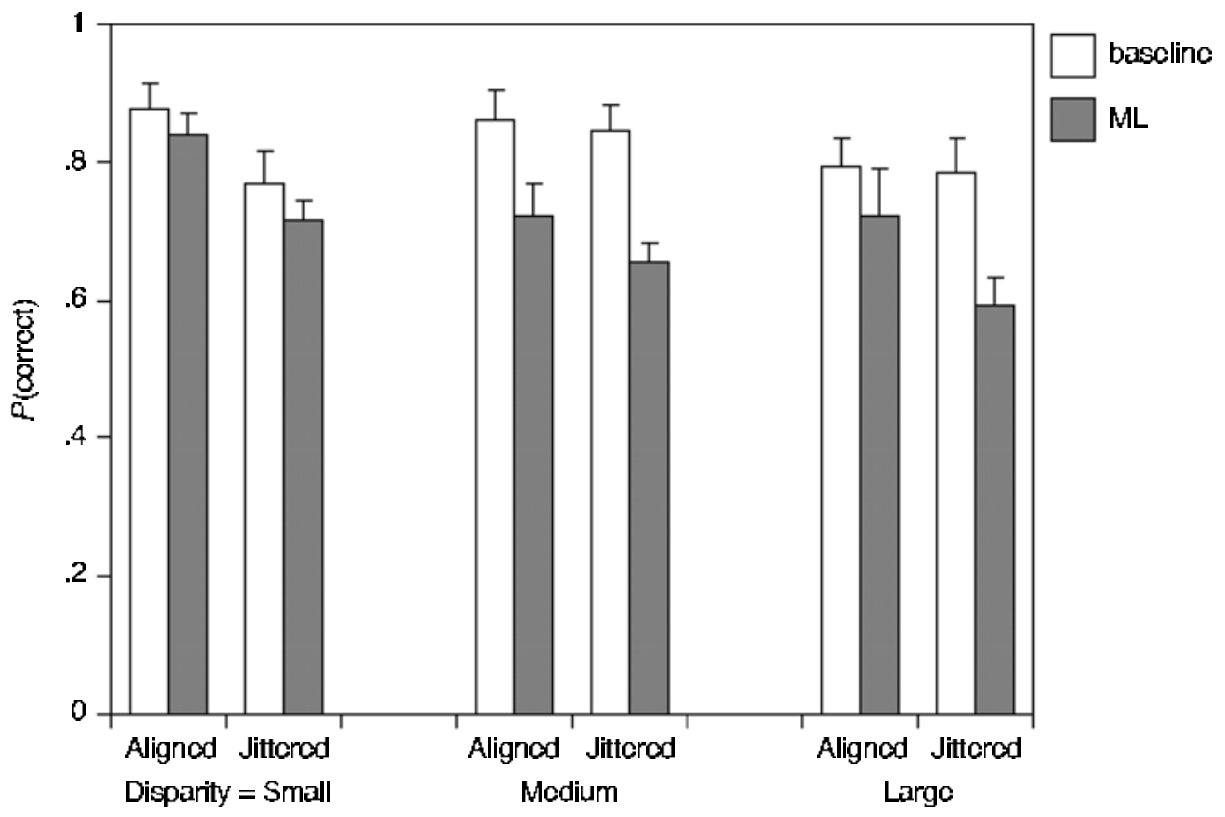

Figure 3. Percentages of correct line judgments for the baseline and the ML conditions as a function of the background pattern in the inattention blocks. The two bars in each group represent performance in the baseline (unfilled bar) and the ML (filled bar) blocks. Left panel, small disparity; center panel, medium disparity; and right panel, large disparity. Error bar $=1 S E$. 
than in the nonjittered condition. This difference was, however, not significant. There was no display type $\times$ jitter interaction $(F<1)$.

\section{Line Judgment: Divided-Attention Condition}

The ML effect shown in the inattention blocks was also observed for the divided-attention blocks. The datacorrect identification of the longer line as a function of the different display conditions - are presented in Figure 4. A 2 (display type: identical vs. ML arrowheads) $\times 3$ (disparity) $\times 2$ (jitter) ANOVA for the line judgment data ${ }^{1}$ revealed main effects of both display type and disparity $\left[F(1,12)=21.918, M S_{\mathrm{e}}=0.035, p<.001\right.$, and $F(2,24)=33.954, M S_{\mathrm{e}}=0.034, p<.001$, respectively]. Accuracy was significantly lower in the ML condition and decreased with increasing disparity. The interaction between the display type and the disparity variables was reliable $\left[F(2,24)=7.306, M S_{\mathrm{e}}=0.024, p<.005\right]$. When the disparity was smallest, the ML illusion was not observed. But as disparity increased, the arrowheads became more apparent, and the illusion became stronger. There appears to be some hint that the jitter variable operated in a direction opposite to that found in the inattention condition: There was a marginally reliable main effect of jitter $\left[F(1,12)=3.308, M S_{\mathrm{e}}=0.013, p<.1\right]$. No other effect was significant.

For the divided-attention blocks, the poorer performance in the ML trials suggests that the lines could not have been processed after the arrowheads. While line judgment performance dropped $13.3 \%$, as compared with the inattention blocks, the decline in the arrowhead task $(27.4 \%)$ was twice as large, as compared with the full-attention blocks. This pattern of results is consistent with Moore and Egeth's (1997) conclusions. The arrowheads were processed preattentively even when the primary task was line discrimination. But there was a bottleneck in the consolidation of these preattentive codes. The codes are fragile, and when they are not immediately consolidated, they quickly degenerate and become unrecoverable (Potter, 1993). The codes obtained from line judgment were consolidated first. If this consolidation stage were allowed to run to completion, the arrowhead codes would probably be irretrievably lost. Some compromise was struck, and there was a switch to consolidating the arrowhead codes even before the line codes were completely consolidated.

\section{Illusion Strength: Inattention and Divided Attention}

Finally, we investigated the relationship between the magnitude of illusion strength and disparity. In the line judgment task, different baseline performance was obtained for the divided-attention and the inattention conditions. Thus, as a measure of illusion strength, the difference score (baseline - ML) was computed and used as the dependent variable in the analysis. Because our

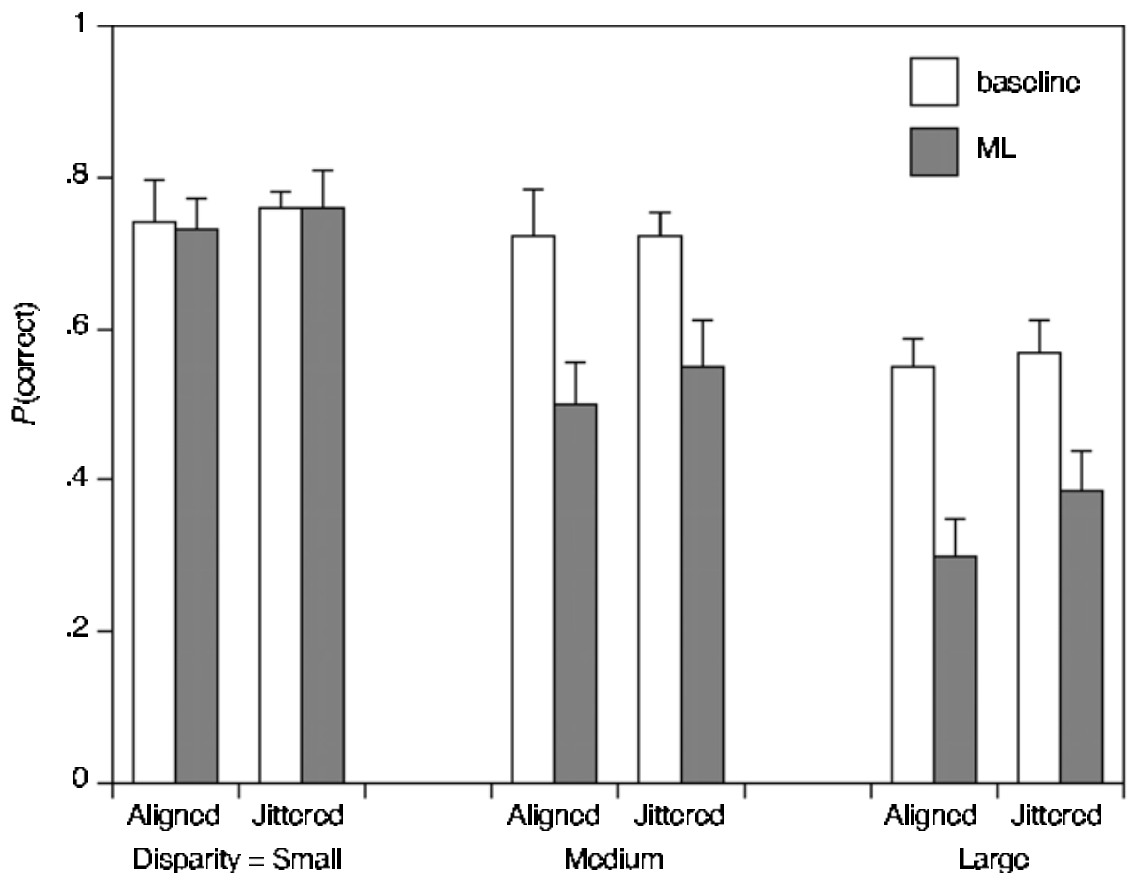

Figure 4. Percentages of correct line judgments for the baseline and the ML conditions as a function of the background pattern in the divided-attention blocks. The two bars in each group represent performance in the baseline (unfilled bar) and the ML (filled bar) blocks. Left panel, small disparity; center panel, medium disparity; and right panel, large disparity. Error bar $=1 S E$. 
previous results showed no ML effect for the smallest disparity condition, only the medium- and large-disparity conditions were considered. A 2 (attention instruction: inattention vs. divided-attention) $\times 2$ (disparity: medium vs. large) $\times 2$ (jitter) ANOVA revealed no significant results other than a marginal effect for the attention instruction variable $\left[F(1,12)=3.818, M S_{\mathrm{e}}=0.035, p<\right.$ $.08] .^{2}$ Critically, the disparity variable produced no reliable effect, nor did it interact with the other variables. This means that as disparity increased, the illusion did not get stronger. But for the arrowhead task, there was a reliable difference in performance between the mediumand the large-disparity conditions $[F(1,12)=80.222$, $\left.M S_{\mathrm{e}}=0.026, p<.001\right]$. What this implies is that although the appearance of the arrowheads could be enhanced by the disparity of the arrowhead and background spots, there was no concomitant strengthening of the ML illusion. So long as the disparity between the spots exceeded some threshold that allowed preattentive grouping by size, the illusion obtained.

\section{CONCLUSION}

The participants succumbed to the illusion even though they reported not having seen the arrowheads. There are two lines of evidence for preattentive grouping. In the inattention blocks, line judgment was the sole task, and the participants did not report having seen any pattern when they were quizzed at the end of those trials. Nevertheless, the poorer performance obtained for the ML conditionsimplied that they must have perceived the arrowheads. The second line of evidence came from the divided-attention trials. In the medium-disparity condition, the participants were essentially guessing the orientation of the arrowheads when the spots were jittered. Yet when the arrowheads were presented, the participants' line discrimination performance was still worse than the baseline for both the divided-attention and the inattention blocks. This result demonstrates a dissociation between perceiving a grouped structure and making a veridical report of the structure. Our results are consistent with Moore and Egeth (1997), demonstrating that the failure to report seeing a grouped pattern does not necessarily imply that grouping did not occur in the first place.

The jitter variable, which did not appreciably affect line judgment in the divided-attention blocks, produced poorer performance in the inattention blocks. This dissociation speaks to Mack et al.'s (1992) observation that forewarning observers that there might be a grouping could influence their perceptual strategies. These results suggest that the grouping that obtained implicitly (of which the participants were more likely to be unaware) influenced line judgments quite differently than that which obtained when recovery of the grouped structure was explicitly demanded.

\section{REFERENCES}

BARChilon Ben-Av, M., \& SAGI, D. (1995). Perceptual grouping by similarity and proximity: Experimental results can be predicted by intensity autocorrelations. Vision Research, 35, 853-866.

BARChilon Ben-Av, M., SAGi, D., \& Braun, J. (1992). Visual attention and perceptual grouping. Perception \& Psychophysics, 52, 277-294.

Coltheart, V. (1999). Fleeting memories: Cognition of brief visual stimuli. Cambridge, MA: MIT Press.

Mack, A., TANG, A., Tuma, R., Kahn, S., \& Rock, I. (1992). Perceptual organization and attention. Cognitive Psychology, 24, 475-501.

Moore, C. M., \& Egeth, H. (1997). Perception without attention: Evidence of grouping under conditions of inattention. Journal of Experimental Psychology: Human Perception \& Performance, 23, 339-352.

Potter, M. C. (1993). Very short-term conceptual memory. Memory \& Cognition, 21, 156-161.

SAGI, D., \& Julesz, B. (1985, June 7). "Where" and "what" in vision. Science, 228, 1217-1219.

Treisman, A. (1986). Properties, parts, and objects. In K. R. Boff, L. Kaufman, \& J. P. Thomas (Eds.), Handbook of perception and human performance (Vol. 2, pp. 35-1 to 35-70). New York: Wiley. WolfE, J. (1999). Inattentional amnesia. In V. Coltheart (Ed.), Fleeting memories: Cognition of brief visual stimuli (pp. 71-94). Cambridge, MA: MIT Press.

\section{NOTES}

1. To be sure that the line judgment task was performed as the primary task, the probabilities of correct line discrimination, given correct arrowhead identification, were compared with the simple probabilities of line judgment. No significant difference was found, suggesting that the line judgment was independent of the arrowhead identification.

2. In the divided-attention blocks, arrowheads enclosed the lines in the baseline trials. In the inattention blocks, they did not. It is possible that the arrowheads could have made the line discrimination task harder (thus, contributing partly to the poorer baseline performance in the dividedattention blocks). The strength of the illusion for the divided-attention blocks possibly was underestimated. The important point, however, is that the illusion still appeared stronger in the divided-attention blocks.

(Manuscript received August 10, 2001; revision accepted for publication July 30, 2002.) 